\title{
Introduction, Classification and Applications of Smart Materials: An Overview
}

\author{
Susmita Kamila \\ Department of Chemistry, East Point College of Engineering and Technology, Bangalore-560 049, India
}

Received 2013-06-13, Revised 2013-07-12; Accepted 2013-07-18

\begin{abstract}
Smart materials are common name for a wide group of different substances. The general feature of all of them is the fact that one or more properties might be significantly altered under controlled condition. The present age is considered to be the smart materials era. Earlier, smart material was defined as the material, which responds to its environments in a timely manner. However, the definition of smart materials has been expanded to the materials that receive, transmit, or process a stimulus and respond by producing a useful effect that may include a signal that the materials are acting upon it. This study focuses on the introduction of smart materials and their classifications. Different applications of smart materials in various fields are also being discussed starting from engineering to the present environment.
\end{abstract}

Keywords: Smart Materials, Actuators, Piezoelectric, External Stimuli

\section{INTRODUCTION}

With the development of material science, many new, high-quality and cost-efficient materials have come into use in various field of engineering. In the last ten decades, the materials became multifunctional and required the optimization of different characterization and properties. With the last evolution, the concept has been driving towards composite materials and recently, the next evolutionary step is being contemplated with the concept of smart materials. Smart materials are new generation materials surpassing the conventional structural and functional materials. These materials possess adaptive capabilities to external stimuli, such as loads or environment, with inherent intelligence. (Rogers, 1988; Rogers et al., 1988) defined smart materials as materials, which possess the ability to change their physical properties in a specific manner in response to specific stimulus input. The stimuli could be pressure, temperature, electric and magnetic fields, chemicals, hydrostatic pressure or nuclear radiation. The associated changeable physical properties could be shape, stiffness, viscosity or damping. Takagi (1990) explained it as intelligent materials that respond to environmental changes at the most optimum conditions and reveal their own functions according to the environment.
Smartness describes self-adaptability, self-sensing, memory and multiple functionalities of the materials or structures. These characteristics provide numerous possible applications for these materials and structures in aerospace, manufacturing, civil infrastructure systems, biomechanics and environment. Self-adaptation characteristics of smart structures are a great benefit that utilizes the embedded adaptation of smart materials like shape memory alloys. By changing their properties, smart materials can detect faults and cracks and therefore are useful as a diagnostic tool. This characteristic can be utilized to activate the smart material embedded in the host material in a proper way to compensate for the fault. This phenomenon is called self-repairing effect.

\subsection{Types}

These materials with one or more property can be significantly altered in a controlled fashion by external stimuli. Thus this material has built-in or intrinsic sensor (s), actuator ( $\mathrm{s}$ ) and control mechanism (s) by which it is capable of sensing a stimulus, responding to it in a predetermined manner and extent, in a short or appropriate time and reverting to its original state as soon as the stimulus is removed. There are a number of types of smart material available and some are discussed below. 
Piezoelectric materials are very common example of such materials where they produce a voltage when stress is applied. Since this effect also applies in the reverse manner, a voltage across the sample will produce stress within the sample. Suitably designed structures made from these materials can therefore be made that bend, expand or contract when a voltage is applied. They can also be used in optical-tracking devices, magnetic heads, dot-matrix printers, computer keyboards, high-frequency stereo speakers, accelerometers, micro-phones, pressure sensors, transducers and igniters for gas grills.

Thermo-responsive materials, either Shape Memory Alloys (SMA) or shape memory polymers, are materials that can hold different shapes at various temperatures. They can be deformed and returned to their original shape by heating. In the process, they generate an actuating force. Shape memory alloy, such as nitinol, an alloy of nickel and titanium, which has a corrosion resistance similar to stainless steel, making it particularly useful for biomechanical applications. Such types of materials can be used in coffee-pot thermostat, super elastic spectacle frames, stents for veins, whereas shape memory polymer has the ability to regain its original shape when heated. These are generally used in biodegradable surgical sutures that will automatically tighten to the correct tension and also in self repairing car bodies that will recover shape on gentle heating after a dent.

Magneto restrictive materials similar to piezoelectrics, respond to only magnetic fields rather than electric. They are typically used in low-frequency, high-power sonar transducers, motors and hydraulic actuators, along with the shape-memory alloy Nitinol, magneto restrictive materials are considered promising candidates for achieving active damping of vibrations.

PH-sensitive materials are the materials that change their colour as a result of changing acidity. This can be applicable for paints that can change colour to indicate corrosion in the metal underneath them.

Chromogenic systems change colour in response to electrical, optical or thermal changes. These include Electrochromic materials, which change their colour or opacity on the application of a voltage. Very common example of such type is Liquid crystal displays. Likewise, photochromic materials, which change colour in response to light. This can be observed in light sensitive spectacles that darken when exposed to bright sunlight. There are also certain paints such as the rmochromic and photochromic paints, which change colour on heating and on exposure to light respectively.
Polymer gels, such as hydrogels, have a cross linked polymer structure inflated with a solvent, such as water; the gel has the ability to swell or shrink (up to 1000 times in volume) due to small changes in, e.g., temperature or $\mathrm{pH}$.

\subsection{Classification}

Smart materials can also be classified into two categories i.e., either active or passive. Fairweather (1998) defined active smart materials as those materials which possess the capacity to modify their geometric or material properties under the application of electric, thermal or magnetic fields, thereby acquiring an inherent capacity to transduce energy. Piezoelectric materials, SMAs, ER fluids and magneto-strictive materials are considered to be the active smart materials and therefore, they can be used as force transducers and actuators. Kumar (1991) showed that SMA has large recovery force, of the order of $700 \mathrm{MPa}\left(10^{5} \mathrm{psi}\right)$, which can be utilized for actuation. Similarly piezoelectric materials, which convert electric energy into mechanical force, are also 'active'.

On the other part, the materials, which are not active, are called passive smart materials. Although smart, they lack the inherent capability to transduce energy. Fiber optic material is a good example of a passive smart material. Such materials can act as sensors but not as actuators or transducers.

\subsection{Applications}

Smart materials find a wide range of applications due to their varied response to external stimuli. The different areas of application can be in our day to day life, aerospace, civil engineering applications and mechatronics to name a few. The scope of application of smart material includes solving engineering problems with unfeasible efficiency and provides an opportunity for creation of new products that generate revenue. Important feature related to smart materials and structures is that they encompass all fields of science and engineering. As far as the technical applications of smart materials is concerned, it involves composite materials embedded with fiber optics, actuators, sensors, MicroElectro Mechanical Systems (MEMSs), vibration control, sound control, shape control, product health or lifetime monitoring, cure monitoring, intelligent processing, active and passive controls, self-repair (healing), artificial organs, novel indicating devices, designed magnets, damping aeroelastic stability and stress distributions. Smart structures are found in automobiles, space systems, fixed-and rotary-wing 
aircrafts, naval vessels, civil structures, machine tools, recreation and medical devices.

The kind of 'smartness' shown by these materials is generally programmed by material composition, special processing, introduction of defects or by modifying the micro-structure, so as to adapt to the various levels of stimuli in a controlled fashion. Like smart structures, the terms 'smart and 'intelligent' are used interchangeably for smart materials. Takagi (1990) defined intelligent materials as the materials which respond to environmental changes at the most optimum conditions and manifest their own functions according to the environment. The feedback functions within the material are combined with properties and functions of the materials.

Smart systems also sense their environment and respond, but are not constructed from a single material. They may incorporate smart materials, but can also be constructed using traditional technology. Pacemakers are a smart system designed to respond to an irregular heart rate with an electrical impulse that regulates it. Vardan and Vardan (2002) reported that smart system refers to a device which can sense changes in its environment and can make anoptimal response by changing its material properties, geometry, mechanical or electromagnetic response. Both the sensor and the actuator functions with their appropriate feedback must be properly integrated. According to (Takagi, 1990), the system could lose its application or could be dangerous if the response is too slow or too fast. Some common smart materials and associated stimulusresponse are listed in figure (Fig. 1).

Smart materials and systems have a wide range of applications. Investment in research and development is driven by factors such as legislation, reducing waste and demand for higher quality of life.

Structures such as buildings, bridges, pipelines, ships and aircraft must be strongly designed and regularly inspected to prevent 'wear and tear' damage from causing disastrous failures. Inspection is expensive and time consuming, while designing to prevent damage can compromise performance. With some modern materials, damage can be internally serious but leave very little surface evidence.

\subsection{Structural Health Monitoring}

Embedding sensors within structures to monitor stress and damage can reduce maintenance costs and increase lifespan. This is already used in over forty bridges worldwide.

\subsection{Self-Repair}

One method in development involves embedding thin tubes containing uncured resin into materials. When damage occurs, these tubes break, exposing the resin which fills any damage and sets. Self-repair could be important in inaccessible environments such as underwater or in space.

\subsection{In the Field of Defense and Space}

Smart materials have been developed to suppress vibrations and change shape in helicopter rotor blades. Shape-memory-alloy devices are also being developed that are capable of achieving accelerated breakup of vortex waves of submarines and similarly different adaptive control surfaces are developed for airplane wings. Besides, present research is on its way to focus on new control technologies for smart materials and design methods for placement of sensors and actuators.

\subsection{In Nuclear Industries}

Smart technology offers new opportunities in nuclear industrial sector for safety enhancement, personal exposure reduction, life-cycle cost reduction and performance improvement. However, the radiation environments associated with nuclear operations represent a unique challenge to the testing, qualification and use of smart materials. However, the use of such smart materials in nuclear facilities requires knowledge about the materials respond to irradiation and how this response is influenced by the radiation dose.

\subsection{In Structural Engineering}

These materials also find application in the field of structural engineering. They are used to monitor the civil engineering structures to evaluate their durability. Not only the smart materials or structures are restricted to sensing but also they adapt to their surrounding environment such as the ability to move, vibrate and demonstrate various other responses. The applications of such adaptive materials involve the capability to control the aero elastic form of the aircraft wing to reduce the pull and improve operational efficiency, to control the vibration of satellites' lightweight structures, Smart structures are also being developed to monitor structural integrity in aircraft and space structures. Effort has been made to investigate certain piezoelectric materials to reduce noise in air conditioners. Besides, in civil engineering, these materials are used to monitor the integrity of bridges, dams, offshore oil-drilling towers where fiber-optic sensors embedded in the structures are utilized to identify the trouble areas. 


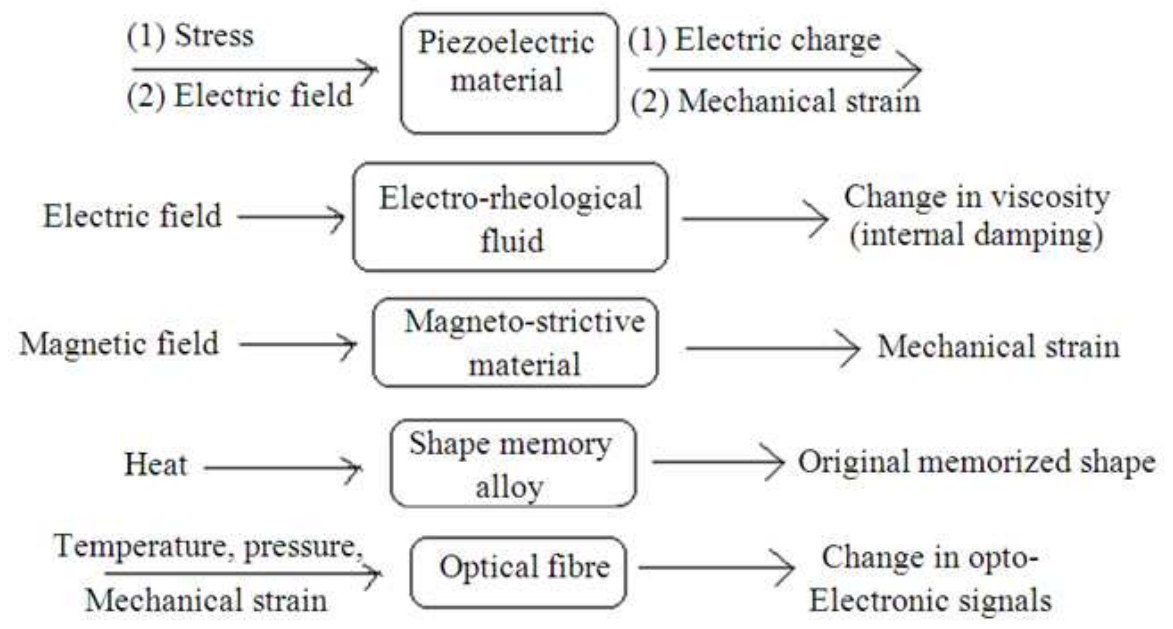

Fig. 1. Common smart materials and associated stimulus-response

\subsection{Biomedical Applications}

In the field of biomedicine and medical diagnostics, still investigations are being carried out. Certain materials like poly-electrolyte gels are being experimented for artificial-muscle applications, where a polymer matrix swollen with a solvent that can expand or contract when exposed to an electric field or other stimulation. In addition, due to biodegradability of these materials, it may make it useful as a drug-delivery system.

\subsection{Reducing Waste}

All over the world, the electronic wastes are the fastest growing components of domestic waste. During disposal and processing of such wastes, hazardous and recyclable materials should be removed first. Manual disassembly is expensive and time consuming but the use of smart materials could help to automate the process. Recently fasteners constructed from shape memory materials are used that can self release on heating. Once the fasteners have been released, components can be separated simply by shaking the product. By using fasteners that react to different temperatures, products could be disassembled hierarchically so that materials can be sorted automatically.

\subsection{Reducing Food Waste}

Food makes up maximum waste among all others. Most of the food grown for consumption is thrown away without consumption due to their reaching of expiry date. These dates are conservative estimates and actual product life may be longer. Manufacturers are now looking for ways to extend product life with packaging by utilizing smart materials.

As food becomes less fresh, chemical reactions take place within the packaging and bacteria build up. Smart labels have been developed that change colour to indicate the presence of an increased level of a chemical or bacteria in it.

Storage temperature has a much greater effect than time on the degradation of most products. Some companies have developed 'time-temperature indicators' that change colour over time at a speed dependent on temperature.

\subsection{Health}

Biosensors made from smart materials can be used to monitor blood sugar levels in diabetics and communicate with a pump that administers insulin as required. However, the human body is a hostile environment and sensors are easily damaged. Some researches on barrier materials are going to protect these sensors.

Now-a-days different companies are developing smart orthopedic implants such as fracture plates that can sense whether bones are healing and communicate data to the surgeon. Small scale clinical trials of such implants have been successful and they could be available within the next five years. Other possible devices include replacement joints that communicate when they become loose or if there is an infection. Current technology limits the response of these devices to transmitting data but in the future, they could respond 
directly by self-tightening or releasing antibiotics. This could reduce the need for invasive surgery.

\subsection{The Ageing Population}

There are now more people aged over 60 in almost every part of universe than children, creating a new market for products that make life easier for the elderly. Many of these could use smart materials and systems to include added functionality. For example, shape memory materials could be used in food packaging that automatically opens on heating for people with arthritis. Smart homes have been developed by researchers for people with dementia that uses sensors to monitor behaviour and to ensure that the resident is safe.

\section{CONCLUSION}

The technology of smart materials by its nature, is a highly interdisciplinary field. Starting from the field of basic sciences such as physics, chemistry, mechanics, computing and electronics it also covers the applied sciences and engineering such as aeronautics and mechanical engineering. This may explain the slow progress of the application of smart structures in engineering systems, even if the science of smart materials is moving very fast. In the present scenario, the most promising technologies for lifetime efficiency and improved reliability include the use of smart materials and structures. Understanding and controlling the composition and microstructure of any new materials are the ultimate objectives of research in this field and is crucial to the production of good smart materials.

\section{REFERENCES}

Fairweather, J.A., 1998. Designing with Active Materials: An Impedance Based Approach. 1st Edn., UMI, New York, pp: 196.

Kumar, S., 1991. Smart materials for accoustic or vibration control. Ph.D. Thesis, Pennsylvania State University.

Rogers, C.A., 1988. Workshop summary. Proceedings U.S. Army Research Office Workshop on Smart Materials, Structures and Mathematical Issues, Sept. 15-16 State University, Technomic Publishing Co., Inc., pp: 1-12.

Rogers, C.A., D.K. Barker, and C.A. Jaeger, 1988. Introduction to Smart Materials and Structures. Proceedings of U.S. Army Research Office Workshop on Smart Materials, Structures and Mathematical Issues, Sept. 15-16, Virginia Polytechnic Institute and State University, Technomic Publishing Co., Inc., pp: 17-28.

Takagi, T., 1990. A concept of intelligent materials. J. Intell. Mater. Syst. Struct., 1: 149-156. DOI: 10.1177/1045389X9000100201

Vardan, V.K. and V.V. Vardan, 2002. Microsensors, Microelectromechanical Systems (MEMS) and electronics for smart structures and systems. Smart Mater. Struct., 9: 953-972. DOI: 10.1088/0964$1726 / 9 / 6 / 327$ 